\title{
Fitossociologia de um fragmento de cerrado sensu stricto na APA do Paranoá, DF, Brasil
}

\author{
Sérgio Lelis Assunção ${ }^{1}$ e Jeanine Maria Felfili1',2
}

Recebido em 01/04/2003. Aceito em 02/06/2004

\begin{abstract}
RESUMO - (Fitossociologia de um fragmento de cerrado sensu stricto na APA do Paranoá, DF, Brasil). Este estudo foi conduzido no Distrito Federal, no Centro Olímpico da Universidade de Brasília. O local é um dos poucos remanescentes de vegetação natural no perímetro urbano de Brasília. O objetivo do trabalho foi estudar a composição florística e a fitossociologia do cerrado sensu stricto, visando obter subsídios para um plano de conservação da área. Foram alocadas aleatoriamente dez parcelas de 20×50m (1.000m²). Foram incluídos na amostragem todos os troncos com diâmetro maior ou igual a 5cm, obtidos a $30 \mathrm{~cm}$ do solo. Foram amostradas 54 espécies distribuídas em 44 gêneros e 30 famílias. A família Leguminosae apresentou o maior número de espécies (9), seguida da Malpighiaceae (4) e Vochysiaseae (4). As espécies com maior Índice do Valor de Importância foram Stryphnodendron adstringens (Mart.) Coville, Styrax ferrugineus Nees \& Mart., Ouratea hexasperma (A. St.-Hill.), Caryocar brasiliense Cambess. e Kielmeyera coriacea (Spreng.) Mart. O índice de Shannon $\left(\mathrm{H}^{\prime}=3,41\right)$ comprovou a alta diversidade da área. A densidade foi de 882 árvores por hectare e a área basal de $9,53 \mathrm{~m}^{2} / \mathrm{ha}$.
\end{abstract}

Palavras-chave: diversidade, fitossociologia, cerrado, Brasil

ABSTRACT - (Phytosociology of a cerrado sensu stricto fragment at the Paranoá Environmental Protection Area, DF, Brazil). This study was conducted in a remnant of cerrado sensu stricto at the Olimpic Center of the University of Brasília, at the Paranoá Environmental Protection Area - DF. The site is one of the few remnants of natural vegetation within the urban perimeter of Brasília. The objective was to investigate the floristic composition and the phytosociology of the cerrado sensu stricto to gather information for a conservation plan. Ten $20 \times 50 \mathrm{~m}\left(1,000 \mathrm{~m}^{2}\right)$ plots were ramdonly located and all woody individuals with dbh equal or greater than $5 \mathrm{~cm}$ at $30 \mathrm{~cm}$ from ground level were identified and measured. A total of 54 species in 44 genera and 30 families were found at the site. Leguminosae had the most species (9), followed by Malpighiaceae (4) and Vochysiaseae (4). The highest importance values were for Stryphnodendron adstringens (Mart.) Coville, Styrax ferrugineus Nees \& Mart., Ouratea hexasperma (A. St.-Hill.), Caryocar brasiliense Cambess. and Kielmeyera coriacea (Spreng.) Mart. Shannon index $\left(H^{\prime}=3.41\right)$ suggests high diversity. Community density was 822 trees per ha with basal area of $9.53 \mathrm{~m}^{2} \cdot \mathrm{ha}^{-1}$.

Key words: diversity, phytosociology, savanna, cerrado, Brazil

\section{Introdução}

O patrimônio natural brasileiro expresso pela extensão continental, pela diversidade e endemismo das espécies biológicas e seu patrimônio genético, bem como pela variedade ecossistêmica dos biomas, apresenta grande relevância mundial.

Entre as mais ricas savanas do mundo, a flora do cerrado brasileiro conta com 6.420 espécies vasculares (Mendonça et al. 1998). O cerrado sensu stricto, que ocupa $70 \%$ do Bioma Cerrado, tem sua paisagem composta por um estrato herbáceo dominado principalmente por gramíneas, e um estrato de árvores e arbustos tortuosos, com ramificações irregulares e retorcidas, variando em cobertura de 10 a $60 \%$ (Eiten 1994). A freqüência de queimadas, a profundidade do lençol freático e os fatores antrópicos têm nítida influência na distribuição das suas espécies arbóreas (Ribeiro \& Walter 1998).

A cobertura original do cerrado brasileiro já foi reduzida em mais de 37\% (Felfili et al. 2002), comprometendo muito a sua biodiversidade. Esse fato, somado à distribuição restrita das espécies (Felfili et al. 1997) e ao pequeno percentual de $1,1 \%$ da área legalmente declarados como Área de Proteção Ambiental e aos 2,5\% declarados como de Preservação Permanente, dão idéia dos riscos de perda das informações sobre a florística da região (Felfili \& Silva Júnior 2001).

O Distrito Federal (DF), localizado na área nuclear do Bioma Cerrado, tem sofrido acelerada ação depredatória dos recursos naturais. Em um período de

\footnotetext{
1 Departamento de Engenharia Florestal, Universidade de Brasília, C. Postal 04357, CEP 70919-970, Brasília, DF, Brasil

2 Autor para correspondência: felfili@unb.br
} 
44 anos após o início de sua ocupação, 73,8\% da cobertura original de Cerrado já foram perdidos (Felfili 2000). As Unidades de Conservação do DF ocupam o total de $42 \%$ de sua área física, mas muitas dessas áreas, inclusive as Áreas de Proteção Ambiental, encontram-se invadidas por edificações ilegais, o que leva a contaminação e assoreamento dos corpos d“água e conseqüente queda da biodiversidade (UNESCO 2000).

Em 14/dezembro/1989, o governo do Distrito Federal assinou o Decreto n. 12.055 criando a Área de Proteção Ambiental do Paranoá, que tem como um dos objetivos, garantir a preservação do ecossistema natural ainda existente na bacia, com os seus recursos bióticos, hídricos, edáficos e aspectos paisagísticos. Dentro dos limites da APA, encontra-se o cerrado sensu stricto estudado, localizado no Centro Olímpico da Universidade de Brasília - UnB, na área conhecida como "matinha do CO". Atualmente o local encontra-se em estado de abandono e vem sofrendo várias ações de degradação, tais como despejos ilegais de lixo e entulho e constantes incêndios. Esses fatos não se justificam, haja vista a riqueza de sua flora, a importância ecológica na proteção da margem do Lago Paranoá e o potencial para realização de pesquisas e programas de Educação Ambiental.

O conhecimento sobre a distribuição e organização da biodiversidade, nas comunidades do Cerrado, são ainda reduzidas. Estas informações são de grande importância para avaliar os impactos antrópicos, planejar a criação de unidades de conservação e a adoção de técnicas de manejo (Felfili \& Silva Júnior 2001).

O objetivo deste trabalho foi realizar o levantamento florístico e fitossociológico da vegetação lenhosa do cerrado sensu stricto do Centro Olímpico da Universidade de Brasília - UnB, a fim de criar subsídios para incentivar a proteção e valorização científica da área.

\section{Material e métodos}

Este estudo foi realizado na APA do Paranoá no Distrito Federal no Centro Olímpico da Universidade de Brasília - UnB em uma área de 110ha conhecida como "matinha do CO”, entre os vértices de

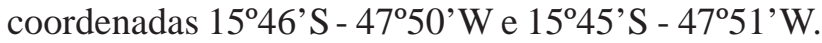
O clima predominante da região corresponde ao tipo Cwa da classificação de Köppen - tropical de Savana. O índice de pluviosidade varia entre 1.400 a $1.450 \mathrm{~mm} /$ ano com a concentração da precipitação pluviométrica no verão. A declividade está entre 2 a $5 \%$ e a altitude entre 1.000 e $1.050 \mathrm{~m}$ (Ferrante et al. 2001). A vegetação da área de estudo é de cerrado sensu stricto sobre Latossolo Vermelho-Escuro. Essa condição é bem representativa das condições de solo do Cerrado, representando 38,65\% dos solos do DF (Haridasan 1994).

O método de amostragem utilizado foi o aleatório (Brena \& Péllico Netto 1997). Conforme a metodologia proposta por Felfili \& Silva Júnior (1988; 1992; 2001) e descrita por Felfili et al. para o cerrado sensu stricto, a amostra totalizou um hectare e foi composta de dez parcelas de $20 \times 50 \mathrm{~m}\left(1.000 \mathrm{~m}^{2}\right)$ distribuídas de modo aleatório nas áreas com cobertura natural de cerrado sensu stricto. Nestas parcelas foram identificados, com seus respectivos nomes científicos, todos os indivíduos lenhosos, com diâmetro igual ou superior a $5 \mathrm{~cm}$, tomado a $0,30 \mathrm{~m}$ de altura do solo. A altura total de cada indivíduo foi também medida. Os dados obtidos em campo foram examinados com o auxílio do programa Mata Nativa. Esse programa realiza cálculos de parâmetros fitossociológicos e foi desenvolvido pela Cientec (Consultoria e Desenvolvimento de Sistemas Ltda.). Foram efetuadas coletas de material botânico fértil nas parcelas e seu entorno pelo perímetro da área, posteriormente depositado no herbário do IBGE.

Para avaliar a abrangência florística do método foi elaborada a curva espécie-área (Kent \& Coker 1992; Felfili \& Venturoli 2000). Foram determinados os intervalos de confiança a $95 \%$ de probabilidade (Brena \& Péllico Netto 1997) para as árvores com diâmetros a partir de $5 \mathrm{~cm}$. Se a curva espécie-área mostra sinais de estabilização e se o erro padrão da média for inferior a $10 \%$, a amostragem pode ser considerada suficiente (Brena \& Péllico Netto 1997).

Foram analisadas a distribuição das alturas, com intervalos de classe de $1 \mathrm{~m}$, e a distribuição dos diâmetros, com intervalos de classe de $5 \mathrm{~cm}$. Para avaliação da diversidade florística da comunidade foi utilizado o Índice de Shannon (Felfili \& Venturoli 2000).

\section{Resultados e discussão}

Suficiência da amostragem - O padrão encontrado na curva espécie-área confirma os padrões conhecidos para o cerrado (Felfili \& Silva Júnior 2001). Pode-se observar (Fig. 1) que a partir de $3.000 \mathrm{~m}^{2}$ de área amostrada começou a haver maior redução no número de novas espécies encontradas, sendo que na sétima parcela, $90 \%$ do total de espécies já havia sido amostrada. Com nove parcelas, a curva estabilizou-se, 


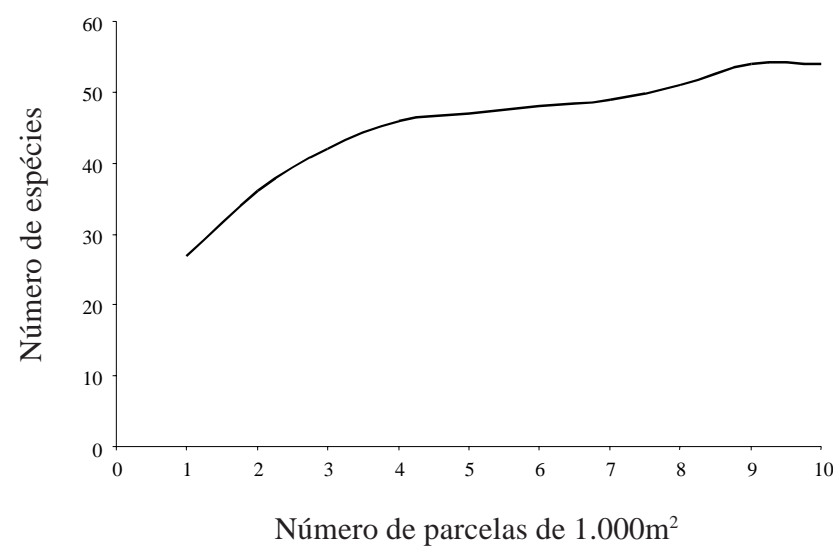

Figura 1. Curva espécie-área do cerrado sensu stricto do Centro Olímpico da Universidade de Brasília (UnB) na APA do Paranoá, Brasília, DF, Brasil.

não havendo mais espécies novas na décima parcela. Isso demonstra que a variabilidade florística foi bem amostrada (Felfili \& Venturoli 2000).

Este cerrado apresentou padrão semelhante ao da Fazenda Água Limpa (Felfili \& Silva Júnior 1992) e ao do Parque Nacional Grande Sertão Veredas, além de três outras localidades na Bahia. Esse padrão indica que as espécies estão distribuídas em mosaicos, ou seja, existe alta similaridade entre áreas próximas de cerrado, ocorrendo a dominância de um grupo de espécies. Para que esta similaridade seja reduzida, são necessárias expressivas diferenciações nas condições do terreno ou elevado distanciamento (Felfili \& Felfili 2001).

O erro padrão (5,92\%) e o intervalo de confiança para a média da densidade $(\mathrm{P}[71,02 \leq \mu \leq 92,98]=0,95)$, por parcela de $1.000 \mathrm{~m}^{2}$, considerando apenas as árvores vivas, satisfazem as exigências de precisão estabelecidas, confirmando a eficiência da amostragem, ou seja, um erro padrão da média, inferior a 10\% com 95\% de confiabilidade.

Composição florística e diversidade - No cerrado sensu stricto do Centro Olímpico da UnB, foram amostradas 54 espécies lenhosas. Esse valor está no intervalo de 50 a 80 espécies normalmente encontradas nos cerrados do DF e na Chapada Pratinha (Felfili \& Silva Júnior 1992; Felfili et al. 1994) e na Chapada do Espigão Mestre do São Francisco, mas foi menor do que a riqueza encontrada na Chapada dos Veadeiros, de 82 a 97 espécies (Felfili \& Silva Júnior 2001).

As 54 espécies estão distribuídas em 44 gêneros e 30 famílias (Tab. 1). Leguminosae teve o maior número de espécies, sendo $16,7 \%$ do total. O gênero Erythroxylum foi o mais diversificado, com três
Tabela 1. Composição florística do componente lenhoso sensu stricto do Centro Olímpico da Universidade de Brasília (UnB) na APA do Paranoá, Brasília, DF.

Família/Espécie

ANNONACEAE

Annona crassifolia Mart.

APOCYNACEAE

Aspidosperma subincanum Mart.

Aspidosperma tomentosum Mart.

ARALIACEAE

Schefflera macrocarpa (Seem.) D.C. Frodin

BIGNONIACEAE

Tabebuia aurea (Manso) Benth. \& Hook. f. ex S. Moore

Tabebuia serratifolia (Vahl) Nicholson

BOMBACACEAE

Eriotheca pubescens (Mart. \& Zucc.) Schott. \& Endl.

Pseudobombax tomentosum (Mart.\& Zucc.) A. Robyns Robyns

CARYOCARACEAE

Caryocar brasiliense Cambess

CELASTRACEAE

Austroplenckia populnea Reiss.

COMPOSITAE

Piptocarpha rotundifolia (Less.) Baker

CONNARACEAE

Connarus suberosus Planchon

EBENACEAE

Diospyros burchellii Hiern.

ERYTHROXYLACEAE

Erythroxylum deciduum A. St.-Hil.

Erythroxylum suberosum A. St.-Hil.

Erythroxylum tortuosum Mart.

GUTTIFERAE

Kielmeyera coriacea (Spreng.) Mart.

HIPPOCRATEACEAE

Salacia crassifolia (Mart. ex Schult.) G. Don

LEGUMINOSAE - CAESALPINOIDEAE

Hymenaea stigonocarpa Mart. ex Hayne

Sclerolobium paniculatum Vog.

LEGUMINOSAE - MIMOSOIDEAE

Dimorphandra mollis Benth.

Enterolobium gummiferum (Mart.) MacBryde

Plathymenia reticulata Benth.

Stryphnodendron adstringens (Mart.) Coville

LEGUMINOSAE - PAPILIONOIDEAE

Bowdichia virgilioides H.B. \& K.

Dalbergia miscolobium Benth.

Machaerium opacum Vog.

LOGANIACEAE

Strychnos pseudoquina A. St.-Hil.

LYTHRACEAE

Lafoensia pacari A. St.-Hil.

MALPIGHIACEAE

Byrsonima coccolobaefolia (Spreng.) H.B. \& K.

Byrsonima crassa Nied.

Byrsonima verbascifolia (L.) DC.

Heteropterys byrsonimifolia A. Juss.

MELASTOMATACEAE

Miconia albicans (Sw.) Triana 
Tabela 1 (continuação)

Família/Espécie

MELASTOMATACEAE

Miconia pohliana Cogn.

MYRSINACEAE

Rapanea guianensis Aubl.

MYRTACEAE

Blepharocalyx salicifolius (H.B. \& K.) Berg

Eugenia dysenterica Mart. ex DC.

Psidium pohlianum Berg

NYCTAGINACEAE

Guapira noxia (Netto) Lund

Neea theifera Oerst.

OCHNACEAE

Ouratea hexasperma (A. St.-Hil.) Baill.

OPILIACEAE

Agonandra brasiliensis Miers ex Benth.

PROTEACEAE

Roupala montana Aubl.

RUBIACEAE

Tocoyena formosa (Cham. \& Schltdl.) K. Schum.

SAPOTACEAE

Pouteria ramiflora (Mart.) Radlk.

Pouteria torta (Mart.) Radlk. ssp. torta

STYRACACEAE

Styrax ferrugineus Nees \& Mart.

SYMPLOCACEAE

Symplocos rhamnifolia A. DC.

VERBENACEAE

Aegiphila lhotzkiana L.

VOCHYSIACEAE

Qualea grandiflora Mart.

Qualea multiflora Mart.

Qualea parviflora Mart.

Vochysia thyrsoidea Pohl

espécies representadas. O total de 63,3\% das famílias e 79,5\% dos gêneros foram representados por apenas uma espécie, refletindo a alta diversidade biológica da área.

Leguminosae tem sido a família mais rica em espécies na maioria dos levantamentos realizados no cerrado (Mendonça et al. 1998; Silva et al. 2002; Weiser \& Godoy 2001). Vochysiaceae, com 7,4\% das espécies amostradas, demonstrou elevada riqueza de espécies, fato verificado em outras áreas do DF, como no cerrado sensu stricto da Fazenda Água Limpa (Felfili \& Silva Júnior 1992) e na Estação Ecológica de Águas Emendadas (Felfili et al. 1994).

Quanto à diversidade de espécies, o índice de Shannon apresentou valor igual a 3,41, indicando alta diversidade e está situado na mesma faixa daqueles encontrados por Felfili et al. (1992; 1994; 2001) em 15 localidades nas Chapadas Pratinha, dos Veadeiros e do Espigão Mestre do São Francisco. Nessas regiões houve variação entre 3,04 a 3,73, sendo que o PARNA Grande Sertão Veredas apresentou valor semelhante ao da área em estudo.

Fitossociologia - A densidade foi de 882 árvores por hectare (Tab. 2) e a área basal de $9,53 \mathrm{~m}^{2} / \mathrm{ha}$. A densidade por hectare apresentou-se menor do que na Estação Ecológica de Águas Emendadas (1.396), APA Gama - Cabeça de Veado (1.394) e no Parque Nacional de Brasília (1.036) (Felfili et al. 1994). A área basal está na média de 5,79 a $10,76 \mathrm{~m}^{2} /$ ha encontrada na Chapada Pratinha (Felfili et al. 1994) e é maior do que a média de 6,19 a $8,33 \mathrm{~m}^{2} /$ ha encontrada na Chapada do Espigão Mestre do São Francisco (Felfili \& Silva Júnior 2001).

As espécies com maiores IVI (índices do valor de importância), em ordem decrescente foram: Stryphnodendron adstringens, Styrax ferrugineus, Ouratea hexasperma, Caryocar brasiliense, Kielmeyera coriacea, Byrsonima coccolobaefolia, Dalbergia miscolobium, Austroplenckia populnea, Eriotheca pubescens, Piptocarpha rotundifolia, Annona crassifolia e Connarus suberosus. Essas 12 espécies representaram 52,2\% do IVI total, 58,5\% da densidade total e $56 \%$ da dominância relativa. Stryphnodendron adstringens, Ouratea hexasperma e Caryocar brasiliense ocorreram também entre as 12 espécies com maior IVI encontradas no levantamento fitossociológico realizado no cerrado sensu stricto da Fazenda Água Limpa (Felfili \& Silva Júnior 1992).

Como essas 22 espécies representam cerca de $40 \%$ do total amostrado, pode-se dizer que esta comunidade caracteriza-se pela existência de poucas espécies dominantes (Andrade et al. 2002) ou seja quande parte da estrutura comunitária do cerrado sensu stricto é formada por poucas espécies de modo que as espécies pouco comuns ou raras apresentam pequena participação na ocupação do espaço. Felfili \& Santos (2002) sugeriram que este padrão de ocupação, encontrado em várias áreas de cerrado e de florestas no Brasil central, seja levado em consideração nos projetos de recuperação de áreas degradadas. Ou seja, pode-se iniciar um processo de recuperação com 10 a 20 espécies dominantes plantadas em maior escala para formar a estrutura da vegetação e complementar o plantio com a maior variedade possível de outras espécies de ocorrência natural na área ou, se houver fontes de propágulos, deixar ocorrer a regeneração natural.

As espécies que apresentaram IVI menor que $10 \%$ do maior valor encontrado $(27,93)$, foram em 
Tabela 2. Fitossociologia do cerrado sensu stricto do Centro Olímpico da Universidade de Brasília (UnB) na APA do Paranoá, Brasília, DF.

\begin{tabular}{|c|c|c|c|c|c|c|c|}
\hline Espécie & $\begin{array}{c}\text { DA } \\
\text { (n/ha) }\end{array}$ & $\begin{array}{l}\text { DR } \\
(\%)\end{array}$ & $\begin{array}{l}\text { FA } \\
(\%)\end{array}$ & $\begin{array}{c}\text { FR } \\
\left(\mathrm{m}^{2} / \mathrm{ha}\right)\end{array}$ & $\begin{array}{c}\text { DoA } \\
(\%)\end{array}$ & DoR & IVI \\
\hline Stryphnodendron adstringens & 98 & 11,11 & 100,00 & 4,00 & 1,222 & 12,82 & 27,93 \\
\hline Styrax ferrugineus & 50 & 5,67 & 100,00 & 4,00 & 0,603 & 6,32 & 15,99 \\
\hline Ouratea hexasperma & 63 & 7,14 & 100,00 & 4,00 & 0,390 & 4,09 & 15,23 \\
\hline Caryocar brasiliense & 22 & 2,49 & 80,00 & 3,20 & 0,867 & 9,10 & 14,79 \\
\hline Kielmeyera coriacea & 59 & 6,69 & 100,00 & 4,00 & 0,295 & 3,09 & 13,78 \\
\hline Byrsonima cocolobaefolia & 45 & 5,10 & 80,00 & 3,20 & 0,422 & 4,42 & 12,72 \\
\hline Dalbergia miscolobium & 37 & 4,20 & 100,00 & 4,00 & 0,322 & 3,38 & 11,57 \\
\hline Austroplenckia populnea & 40 & 4,54 & 60,00 & 2,40 & 0,263 & 2,76 & 9,69 \\
\hline Eriotheca pubescens & 21 & 2,38 & 70,00 & 2,80 & 0,369 & 3,87 & 9,06 \\
\hline Piptocarpha rotundifolia & 31 & 3,51 & 80,00 & 3,20 & 0,194 & 2,04 & 8,75 \\
\hline Annona crassifolia & 26 & 2,95 & 90,00 & 3,60 & 0,196 & 2,06 & 8,61 \\
\hline Connarus suberosus & 24 & 2,72 & 90,00 & 3,60 & 0,194 & 2,03 & 8,35 \\
\hline Rapanea guianensis & 22 & 2,49 & 80,00 & 3,20 & 0,234 & 2,45 & 8,15 \\
\hline Pouteria ramiflora & 21 & 2,38 & 50,00 & 2,00 & 0,331 & 3,48 & 7,86 \\
\hline Diospyros burchellii & 25 & 2,83 & 70,00 & 2,80 & 0,179 & 1,87 & 7,51 \\
\hline Pouteria torta & 21 & 2,38 & 60,00 & 2,40 & 0,174 & 1,83 & 6,61 \\
\hline Blepharocalyx salicifolius & 8 & 0,91 & 40,00 & 1,60 & 0,390 & 4,09 & 6,60 \\
\hline Roupala montana & 10 & 1,13 & 60,00 & 2,40 & 0,225 & 2,36 & 5,90 \\
\hline Aspidosperma tomentosum & 13 & 1,47 & 70,00 & 2,80 & 0,088 & 0,92 & 5,19 \\
\hline Qualea grandiflora & 12 & 1,36 & 60,00 & 2,40 & 0,119 & 1,25 & 5,01 \\
\hline Erythroxylum suberosum & 18 & 2,04 & 50,00 & 2,00 & 0,069 & 0,72 & 4,76 \\
\hline Sclerolobium paniculatum & 11 & 1,25 & 60,00 & 2,40 & 0,096 & 1,01 & 4,65 \\
\hline Schefflera macrocarpa & 11 & 1,25 & 70,00 & 2,80 & 0,049 & 0,51 & 4,56 \\
\hline Enterolobium gummiferum & 9 & 1,02 & 60,00 & 2,40 & 0,099 & 1,03 & 4,45 \\
\hline Machaerium opacum & 7 & 0,79 & 50,00 & 2,00 & 0,143 & 1,50 & 4,29 \\
\hline Dimorphandra mollis & 12 & 1,36 & 50,00 & 2,00 & 0,077 & 0,81 & 4,17 \\
\hline Qualea parviflora & 7 & 0,79 & 40,00 & 1,60 & 0,159 & 1,67 & 4,06 \\
\hline Agonandra brasiliensis & 13 & 1,47 & 20,00 & 0,80 & 0,156 & 1,66 & 3,94 \\
\hline Miconia pohliana & 8 & 0,91 & 30,00 & 1,20 & 0,148 & 1,55 & 3,66 \\
\hline Tabebuia aurea & 8 & 0,91 & 40,00 & 1,60 & 0,068 & 0,72 & 3,22 \\
\hline Salacia crassifolia & 9 & 1,02 & 30,00 & 1,20 & 0,072 & 0,76 & 2,98 \\
\hline Strychnos pseudoquina & 2 & 0,23 & 20,00 & 0,80 & 0,169 & 1,77 & 2,8 \\
\hline Miconia albicans & 7 & 0,79 & 10,00 & 0,40 & 0,120 & 1,26 & 2,46 \\
\hline Byrsonima verbascifolia & 6 & 0,68 & 30,00 & 1,20 & 0,035 & 0,36 & 2,24 \\
\hline Tabebuia serratifolia & 3 & 0,34 & 20,00 & 0,80 & 0,074 & 0,78 & 1,92 \\
\hline Lafoensia pacari & 3 & 0,34 & 30,00 & 1,20 & 0,032 & 0,33 & 1,87 \\
\hline Aegiphila lhotzkiana & 4 & 0,45 & 30,00 & 1,20 & 0,015 & 0,16 & 1,81 \\
\hline Hymenaea stigonocarpa & 4 & 0,45 & 20,00 & 0,80 & 0,031 & 0,32 & 1,58 \\
\hline Bowdichia virgilioides & 4 & 0,45 & 20,00 & 0,80 & 0,029 & 0,30 & 1,56 \\
\hline Erythroxylum tortuosum & 4 & 0,45 & 20,00 & 0,80 & 0,017 & 0,18 & 1,44 \\
\hline Erythroxylum deciduum & 3 & 0,34 & 20,00 & 0,80 & 0,017 & 0,18 & 1,32 \\
\hline Symplocos rhamnifolia & 2 & 0,23 & 20,00 & 0,80 & 0,014 & 0,15 & 1,18 \\
\hline Plathymenia reticulata & 1 & 0,11 & 10,00 & 0,40 & 0,045 & 0,47 & 0,99 \\
\hline Heteropterys byrsonimifolia & 1 & 0,11 & 10,00 & 0,40 & 0,044 & 0,46 & 0,98 \\
\hline Byrsonima crassa & 3 & 0,34 & 10,00 & 0,40 & 0,015 & 0,16 & 0,9 \\
\hline Pseudobombax tomentosum & 2 & 0,23 & 10,00 & 0,40 & 0,021 & 0,22 & 0,85 \\
\hline Guapira похіа & 1 & 0,11 & 10,00 & 0,40 & 0,026 & 0,27 & 0,79 \\
\hline Vochysia thyrsoidea & 1 & 0,11 & 10,00 & 0,40 & 0,018 & 0,19 & 0,71 \\
\hline Qualea multiflora & 1 & 0,11 & 10,00 & 0,40 & 0,015 & 0,16 & 0,67 \\
\hline Neea theifera & 1 & 0,11 & 10,00 & 0,40 & 0,005 & 0,05 & 0,57 \\
\hline Aspidosperma subincanum & 1 & 0,11 & 10,00 & 0,40 & 0,004 & 0,04 & 0,55 \\
\hline Eugenia dysenterica & 1 & 0,11 & 10,00 & 0,40 & 0,004 & 0,04 & 0,55 \\
\hline Psidium pohlianum & 1 & 0,11 & 10,00 & 0,40 & 0,003 & 0,03 & 0,54 \\
\hline Tocoyena formosa & 1 & 0,11 & 10,00 & 0,40 & 0,002 & 0,02 & 0,54 \\
\hline Árvores mortas & 64 & 7,26 & 100,00 & 4,00 & 0,562 & 5,89 & 17,15 \\
\hline Totais & 882 & 100,00 & 2500,00 & 100,00 & 9,530 & 100,00 & 300,00 \\
\hline
\end{tabular}


ordem decrescente: Miconia albicans, Byrsonima verbascifolia, Tabebuia serratifolia, Lafoensia pacari, Aegiphila Ihotzkiana, Hymenaea stigonocarpa, Bowdichia virgilioides, Erythroxylum tortuosum, Erythroxylum deciduum, Symplocos rhamnifolia, Plathymenia reticulata, Heteropterys byrsonimifolia, Byrsonima crassa, Pseudobombax tomentosum, Guapira noxia, Vochysia thyrsoidea, Qualea multiflora, Neea theifera, Aspidosperma subincanum, Eugenia dysenterica, Psidium pohlianum e Tocoyena formosa e podem ser consideradas como pouco comuns na área de estudo.

A espécie Stryphnodendron adstringens foi a que apresentou maior dominância (12,8\% do total), densidade (11,1\% do total) e IVI (9,1\% do total). No cerrado sensu stricto da Fazenda Água Limpa, Felfili \& Silva Júnior (1992) encontraram IVI de 9,7\% do total amostrado, para esta espécie. Em outras áreas do DF, a média encontrada foi de 4,1\% a 6,5\% (Felfili et al. 1994).

As espécies que apresentaram maiores densidades foram: Stryphnodendron adstringens, Ouratea hexasperma, Styrax ferrugineus e Kielmeyera coriacea. Essas representaram 30,6\% do total de espécies. Ouratea hexasperma apresenta pequeno porte (Mendonça et al. 1998), o que pode influenciar sua alta densidade (Andrade et al. 2002). Kielmeyera coriacea apresenta ampla distribuição no DF (Felfili et al. 1994) e ocorre freqüentemente na Fazenda Água Limpa (Barros 2002).

As espécies com maior dominância foram Stryphnodendron adstringens (12,8\% do total), Caryocar brasiliense (9,1\% do total) e Styrax ferrugineus (6,3\% do total). Essas representaram 28,2\% da dominância relativa. Caryocar brasiliense e Styrax ferrugineus destacaram-se também na Estação Ecológica de Águas Emendadas, APA Gama - Cabeça de Veado e no Parque Nacional de Brasília (Felfili et al. 1994). A variação da dominância relativa nas referidas áreas, cujos levantamentos foram realizados com metodologia similar (Felfili et al. 1994), foi para o Caryocar brasiliense de $6,4 \%$ a $7,3 \%$ e para o Styrax ferrugineus, o intervalo foi de 3,6\% a $6,9 \%$ indicando que estas são espécies dominantes no cerrado sensu stricto das Unidades de Conservação do Distrito Federal. É posivo o fato de parcelas significativas de suas populações estarem representadas nas maiores Unidades de Conservação do Distrito Federal, sendo ameaça à sua sustentabilidade o isolamento entre as unidades (UNESCO 2000), tornando importantes fragmentos como este que podem funcionar como trampolins de biodiversidade (stepping stones), possibilitando o fluxo de pólen e a dispersão especialmente pelo vento e pela avifauna (Felfili 2003).

Os indivíduos mortos ocorreram em todas as parcelas, com densidade relativa elevada de 7,3\% e o segundo maior IVI (5,7\% do total). Segundo Felfili e Silva Júnior (1992), isso pode indicar a ocorrência de recentes distúrbios. A densidade relativa de árvores mortas na Chapada Pratinha e na Chapada do Espigão Mestre do São Francisco apresentou variações entre 4 a 13,7\%, com a maioria das áreas em cerca de 7\% (Felfili et al. 1994; Felfili \& Silva Júnior 2001).

Distribuição das alturas e diâmetros - A distribuição diamétrica da comunidade em estudo apresentou a forma de J-invertido (Fig. 2). A área apresenta principalmente indivíduos jovens, pois mais de 59\% do total amostrado apresentou diâmetros inferiores a $10 \mathrm{~cm}$, e alturas menores que $4 \mathrm{~m}$ (Fig. 3). O diâmetro máximo encontrado foi de $45 \mathrm{~cm}$, pertencente a um indivíduo da espécie Caryocar brasiliense. A maior altura encontrada foi de $10,5 \mathrm{~m}$, pertencente ao único indivíduo amostrado da espécie Vochysia thyrsoidea.

No estudo realizado na Chapada do Espigão Mestre do São Francisco (Felfili \& Silva Júnior 2001), as distribuições diamétricas das quatro áreas de cerrado sensu stricto amostradas apresentaram o formato de J-invertido. A percentagem de indivíduos com até $10 \mathrm{~cm}$ de diâmetro foi maior que $60 \%$ nos municípios de São Desidério (BA), Correntina (BA) e no Parque Nacional Grande Sertão Veredas (MG). No município de Formosa do Rio Preto, mais de 50\% dos indivíduos possuíam menos que $10 \mathrm{~cm}$ diâm. Essas áreas apresentaram 95\% dos indivíduos com diâmetros até $25 \mathrm{~cm}$ padrão confirmado no cerrado sensu stricto do

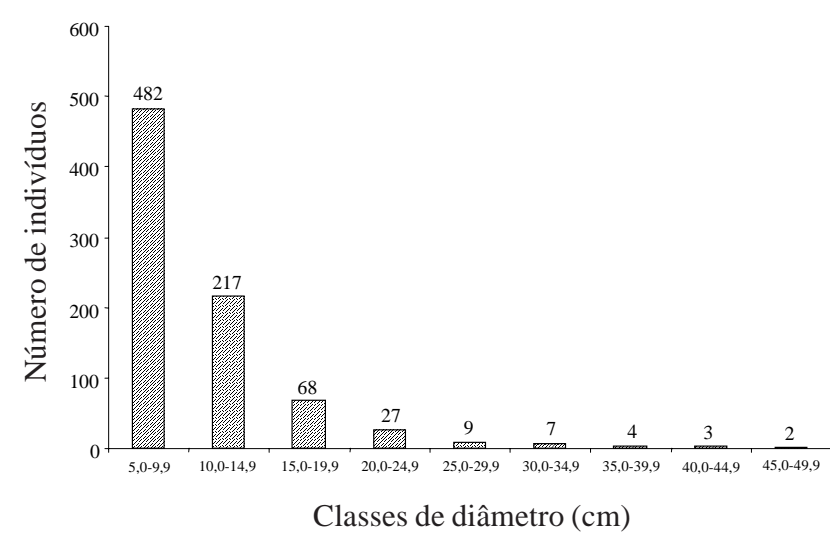

Figura 2. Distribuição dos indivíduos por classes de diâmetro no cerrado sensu stricto do Centro Olímpico da Universidade de Brasília (UnB) na APA do Paranoá, Brasília, DF, Brasil. 


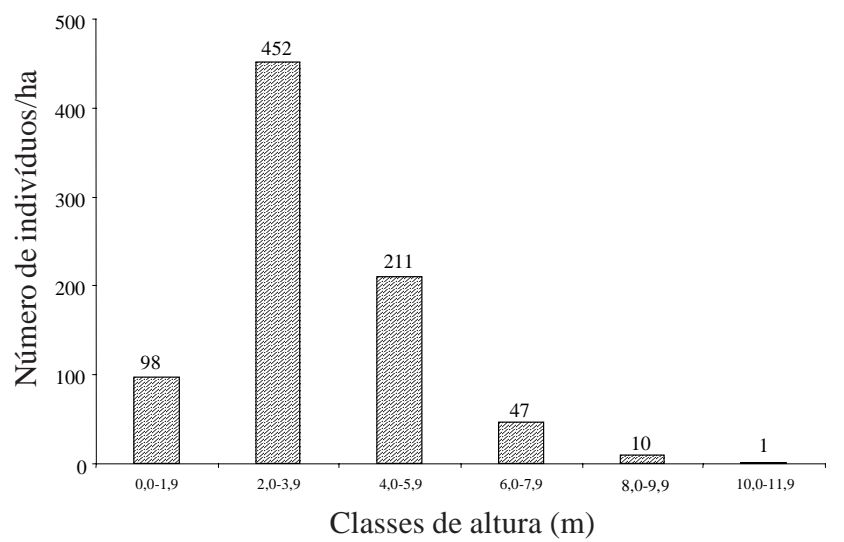

Figura 3. Distribuição dos indivíduos por classes de altura no cerrado sensu do Centro Olímpico da Universidade de Brasília (UnB) na APA do Paranoá, Brasília, DF, Brasil.

Centro Olímpico, com mais de 90\% dos indivíduos atingindo esse limite.

A elevada velocidade com que esse tipo de cerrado vem sendo degradado no Distrito Federal e a alta diversidade e riqueza de espécies do cerrado do Centro Olímpico sugerem que este seja considerado para a implantação de uma unidade de conservação de uso indireto.

O formato de J-invertido resultante da distribuição diamétrica indica que a comunidade estudada é autoregenerativa, caso não seja intensivamente perturbada. O elevado percentual de árvores mortas é um indicativo de perturbações na área, reforçando a necessidade de maior proteção contra incêndios e despejo de lixo e entulhos.

Espécies de elevado valor extrativista como Stryphnodendron adstringens (barbatimão) e Caryocar brasiliense (pequi) são abundantes na área, elevando o seu valor para conservação, especialmente considerando o grau de ameaça dessas espécies pelas pressões extrativistas e pela destruição de seus ambientes naturais.

\section{Referências bibliográficas}

Andrade, L.A.Z.; Felfili, M.J. \& Violatti, L. 2002. Fitossociologia de uma área de cerrado denso na RECOR-IBGE, Brasília-DF. Acta Botanica Brasilica 16(2): 225-240.

Barros, M.A.G. 2002. Floração sincrônica e sistemas reprodutivos em quatro espécies de Kielmeyera Mart. (Guttiferae). Acta Botanica Brasilica 16(1): 113-122.

Brena, D.A. \& Péllico Neto, S. 1997. Inventário florestal. Curitiba.

Eiten, G. 1994. Vegetação do Cerrado. Pp. 17-73. In: M.N. Pinto (org.). Cerrado: caracterização, ocupaçãe perspectivas. Brasília, Ed. UnB/SEMATEC.
Felfili, J.M.\& Silva Júnior, M.C. 1992. Floristic composition, phytosociology and comparison of cerrado and gallery forests at Fazenda Água Limpa, Federal District, Brazil. Pp. 393-415. In: P.A. Furley; J.A. Proctor \& J.A. Ratter. Nature and dynamics of forest-savanna boudaries. London, Chapman \& Hall.

Felfili, J.M.; Filgueiras, T.S.; Haridasan, M.; Silva Júnior, M.C.; Mendonça, R. \& Rezende, A.V. (eds.). 1994. Projeto biogeografia do bioma cerrado: Vegetação e solos. Caderno de Geociências do IBGE 12: 75-166.

Felfili, J.M.; Silva Júnior, M.C.; Rezende, A.V.; Nogueira, P.E.; Walter, B.M.T.; Silva, M.A. \& Encinas, J.I. 1997. Comparação florística e fitossociológica do Cerrado nas Chapadas Pratinha e dos Veadeiros. Pp. 6-11. In: L. Leite \& C.H. Saito (eds.). Contribuição ao conhecimento ecológico do cerrado. Brasília, Ed. Universidade de Brasília.

Felfili, J.M. \& Venturoli, F. 2000. Tópicos em análise de vegetação. Comunicações técnicas florestais, v.2, n.2. Universidade de Brasília, Departamento de Engenharia florestal.

Felfili, J.M. 2000. Perda da diversidade. In: UNESCO. Vegetação no Distrito Federal: tempo e espaço. Brasília: 33-34.

Felfili, J.M. \& Felfili, M.C. 2001. Diversidade Alfa e Beta no cerrado sensu stricto da Chapada Pratinha, Brasil. Acta Botanica Brasilica 15(2): 243-254.

Felfili, J.M.\& Silva Júnior, M.C. (orgs.). 2001. Biogeografia do Bioma Cerrado: estudo fitofisionômico da Chapada do Espigão Mestre do São Francisco.

Felfili, J.M.; Nogueira, P.E.; Silva Júnior, M.C.; Marimon, B.S. \& Delitti, W.B.C. 2002. Composição florística e fitossociologia do cerrado sentido restrito no município de Água Boa, MT. Acta Botanica Brasilica 16(1): 103-112.

Ferrante, J.E.T.; Netto, B.N. \& Rancan, L. 2001. Meio físico. In: F.O. Fonseca (org.). Olhares sobre o lago Paranoá. Brasília, Secretaria do Meio Ambiente e Recursos Hídricos.

Haridasan, M. 1994. Solos. In: M.N. Pinto (org.). Cerrado: caracterização, ocupação e perspectivas. Brasília, Ed. UnB/SEMATEC.

Mendonça, R.C.; Felfili, J.M.; Walter, B.M.T.; Silva Júnior, M.C.; Rezende, A.V.; Filgueiras, T.S. \& Nogueira, P.E. 1998. Flora vascular do cerrado. Pp. 289-556. In: S.M. Sano \& S.P. Almeida. Cerrado, Ambiente e flora. Planaltina, EMBRAPA CPAC.

Ribeiro, J.F. \& Walter, B.M.T. 1998. Fitofisionomias do bioma Cerrado. Pp. 87-167. In: S.M. Sano \& S.P. Almeida. Cerrado, Ambiente e flora. Planaltina, EMBRAPA CPAC.

Silva, L.O.; Costa, D.A.; Espírito Santo Filho, K.; Ferreira, H.D. \& Brandão, D. 2002. Levantamento florístico e fitossociológico em duas áreas de cerrado sensu stricto no Parque Estadual da Serra de Caldas Novas, Goiás. Acta Botanica Brasilica 16(2): 43-53.

UNESCO. 2000. Vegetação no Distrito Federal: tempo e espaço.

Weiser, V.L. \& Godoy, S.A.P. 2001. Florística em um hectare de cerrado stricto sensu na ARIE-Cerrado Pé-de-Gigante, Santa Rita do Passa Quatro, SP. Acta Botanica Brasilica 15(2): 201-212.

Versão eletrônica do artigo em www.scielo.br/abb 